\title{
Swimming Helps Elderly Population to Improve Mental Speed and Attention
}

\author{
Sakharayapatna Vasegowda*
}

\section{Dr. Sakharayapatna Vasegowda*}

Assistant professor, Department of Physiology, Sri Siddartha Institute of Medical Science and Research Centre, Karnataka, INDIA.

\section{Correspondence}

\section{Dr Varsha SV}

Department of Physiology, SS Institute of Medical Science and Research Centre, Karnataka, INDIA.

Phone: +919481581623

Email: drvarshasv@gmail.com

History

- Submission Date: 13-10-2018;

- Review completed: 25-11-2018;

- Accepted Date: 16-12-2018.

DOI : 10.5530/ijcep.2018.5.4.22

\section{Copyright}

(C) 2018 Phcog.Net. This is an openaccess article distributed under the terms of the Creative Commons Attribution 4.0 International license.

\begin{abstract}
Background and Aim: Swimming is an ideal physical activity for the elderly population because of its low impact on joints and minimal risk of injury. Previous studies have proven that swimming has beneficial effect on skeletal and cardiovascular health. But studies to assess effects of swimming on cognitive health of elderly are lacking. Methods: This study was undertaken to assess and compare the level of cognition among elderly swimmers and non swimmers. 50 elderly subjects who regularly swim and 50 elderly non swimmers were recruited. Digit symbol substitution test and Digit vigilance test were done to assess the mental speed and attention of the subjects. Results: Results were statistically analyzed using students t test to compare between the groups. Swimmers took less time to complete both Digit symbol substitution test and Digit vigilance test than non swimmers. Conclusion: Elderly swimmers had better mental speed and attention compared to non swimmers. Key words: Swimming, Aerobic exercise, Elderly, Cognition, Neurogenesis.
\end{abstract}

\section{INTRODUCTION}

Chronological aging or senescence, is associated with an increased risk of chronic conditions and diseases such as cognitive impairment, cardiovascular disease and metabolic syndrome. Due to prolonged life expectancy, age-related diseases have increased in alarming proportions in recent decades. An increasing body of studies have suggested that lifestyle factors have a significant impact on how well people age. Three lifestyle factors can play a significant role in slowing the rate of cognitive decline and preventing dementia: a socially integrated network, cognitive leisure activity and regular physical activity. ${ }^{[1]}$

Swimming is an ideal physical activity for the elderly population because of its low impact on joints and minimal risk of injury. Swimming is also a complete workout, incorporating the entire body and utilizing all muscles groups. ${ }^{[2]}$

To the best of our knowledge, only one study was designated to explicitly test the specific relationship between water aerobics and cognitive improvements in older adults. ${ }^{[3]}$ In this study, Hawkins and collaborators examined the influence of a 10 -week program of basic swimming skills on variations of two experimental tasks involving processing speed. They found that subjects who trained in water aerobics showed significantly better results than the control group.

But recently no studies have been done to study the effect of swimming on the age related cognitive decline. So this study was undertaken to study the effect of swimming on mental speed and attention of elderly population who are at the verge of cognitive decline.

\section{MATERIALS AND METHODS}

Tests were administered to 100 subjects (elderly, male, aged $>60$ years). Among them 50 subjects who regularly swim at least $30 \mathrm{~min}$ for 4 days a week from past $1 \mathrm{yr}$ were recruited. Only male subjects volunteered, so because of lack of subjects, female subjects were not included in the study. Subjects with untreated cataract, refractory errors and history of medical/surgical illness, Alcohol consumption and smoking were excluded from the study. Then $50 \mathrm{el}-$ derly non swimmers who were matched for age and gender with swimmers were recruited. And those who perform other regular physical activity were also dropped from the study.

Institutional ethical clearance was obtained prior to the study. Participants' written informed consent was obtained after explaining about the study protocol and then subjects were asked to relax for $30 \mathrm{~min}$. The participants were then tested for two domains of cognition - speed and attention using Digit symbol substitution test and Digit vigilance test respectively in a noise free surrounding. ${ }^{[4]}$

Digit symbol substitution test: A sheet containing numbers 1-9 randomly arranged in four rows of 25 squares each was provided to the subject. The subject was made to substitute each number with symbol using a number-symbol key given on top of the page. The time taken to complete the test formed the score. Digit Symbol Substitution tests the visuomotor coordination, motor persistence, sustained attention and response speed. Rapid information processing is required in order to substitute the symbols accurately and quickly. 
Digit vigilance test: In this test a sheet was provided which contained numbers 1 to 9 randomly ordered and placed in rows. There were 30 digits per row and 50 rows on the sheet. The digits were closely packed on the sheet. The subject had to focus on the target digits 6 and 9 amongst other distracter digits and had to cancel the digits as fast as possible without missing the targets or cancelling wrong numbers. Inability to sustain and focus attention levels leads to both increased time to complete the test as well as errors. The time taken to complete the test formed the score.

The Descriptive statistics were used, i.e. mean and Standard Deviation (SD) for describing the parameters. The data was analyzed using student t-test to compare between the groups. The difference was considered statistically significant when $P £ 0.05$. SPSS V.11.0 was used for analysis of data.

\section{RESULTS}

The two groups did not differ significantly with respect to demographic characteristics. Results of present study show significant difference between elderly swimmers and non swimmers in both the tests for. Wherein nonswimmers had lower cognitive ability compared to the swimmers. Elderly swimmers were able substitute number with symbols in less duration $(219.29 \pm 19.6)$ than non swimmers $(231.8 \pm 15.4)$, which was statistically significant ( $P$ value- 0.0401$)$ which is represented in Table 2.

In Digit vigilance test which was used to assess attention elderly swimmers performed better by completing the test in less duration of time $(513 \pm 11.79)$ compared to nonswimmers $(525 \pm 12.81)$, represented in Table 2 .

\section{DISCUSSION}

Cerebral and cognitive decline, as a function of aging, represents a predominant cause of autonomy loss in aging populations.

A meta-analysis by Colcombe and Kramer ${ }^{[5]}$ showed that the effect size of the positive effects of chronic exercise is significantly smaller on information processing speed than on executive functions.

Several studies support the notion that physical activity is a significant moderator of age-related cognitive decline. In cross-sectional studies, age-related differences in cognitive performance observed when older adults are compared to younger participants are reduced if the comparisons involved higher-fit individuals rather than sedentary older adults. ${ }^{[6-10]}$ As a whole, these cross-sectional studies suggest that cardio respiratory fitness is associated with more efficient cognitive functions.

Table 1: Baseline characteristic of the study participants.

$\begin{array}{cccc} & \begin{array}{c}\text { Swimmers } \\ (\boldsymbol{n}=50)\end{array} & \begin{array}{c}\text { Non swimmers } \\ (\boldsymbol{n}=50)\end{array} & \text { Total }(\boldsymbol{n}=100) \\ \text { Age (years) } & 67.1 \pm 6.1 & 67.3 \pm 4.7 & 66.2 \pm 5.4 \\ \text { Height }(\mathbf{c m}) & 169.2 \pm 7.3 & 167.2 \pm 6.5 & 168.8 \pm 9.2 \\ \text { Body weight }(\mathbf{K g}) & 74.1 \pm 11.3 & 76.7 \pm 6.5 & 75.1 \pm 6.5 \\ \text { BMI }(\mathbf{K g} / \mathbf{m} 2) & 24.6 \pm 2.0 & 25.7 \pm 1.7 & 25.2 \pm 1.8\end{array}$

Table 2: Comparison of cognitive tests between elderly swimmers and non swimmers.

\begin{tabular}{|cccc|}
\hline Cognitive tests & Swimmers & Non Swimmers & $P$ Value \\
\hline DSST(sec) & $219.29 \pm 19.6$ & $231.8 \pm 15.4$ & $0.0401^{*}$ \\
DVT(sec) & $513 \pm 11.79$ & $525 \pm 12.81$ & $0.0036^{* * *}$
\end{tabular}

Note: DSST - Digit Symbol Substitution Test, Digit Vigilance Test ${ }^{\star} P$ value $<0.05$ significant, ${ }^{* *} P$ value $<0.005$ highly significant.
In the present study the comparison was done among Elderly population, between swimmers and nonswimmers. The results show that Elderly swimmers performed significantly better than non swimmers in terms of both speed and attention.

In Kramer et al. ${ }^{[11]}$ study, older adults who completed a six-month aerobic training program (walking) showed a significant improvement in cognitive performance unlike those who completed a stretching program. Cognitive improvement was greater in tasks that tapped attention and was correlated with improvement in $\mathrm{VO}_{2}$ max. These results suggest that aerobic exercise may be an important cardiac and brain protective factor as people age. The greater improvement induced by aerobic training in mental processing speed and attention also been confirmed by several meta-analyses.

To the best of our knowledge, only one study was designated to explicitly test the specific relationship between water aerobics and cognitive improvements in older adults. ${ }^{[3]}$ In this study, Hawkins and collaborators examined the influence of a 10 -week program of basic swimming skills on variations of two experimental tasks involving processing speed. They found that subjects who trained in water aerobics showed significantly better results than the control group

Better mental speed and attention among swimmers than nonswimmers may be attributed to the neuro-protective effect of swimming, an aerobic exercise. It promotes neurogenesis by increasing the production of neurotrophic factors (compounds which promote the growth or survival of neurons), such as Brain-derived Neurotrophic Factor (BDNF), Insulin-like Growth Factor 1 (IGF-1) and vascular endothelial growth factor (VEGF). ${ }^{[12]}$

\section{CONCLUSION}

Swimming protects elderly population to improve mental speed and attention. Including swimming as a part of day to day activity may help in reducing age related cognitive decline.

\section{LIMITATION OF THE STUDY}

Evidence for the effectiveness of swimming on cognition was demonstrated in this study, even though it is a cross sectional study, swimming may be a useful tool for the all age groups, to be practiced daily basis, for psychological health and to reduce age related cognitive decline. Although our findings are promising, Our study was a cross sectional study; there is a need for a controlled trial in future to clearly study the effect of swimming on cognition and age related cognitive decline among elderly.

\section{REFERENCES}

1. Louis B, Kirk IE, Liu-Ambrose T. A Review of the Effects of Physical Activity and Exercise on Cognitive and Brain Functions in Older Adults. Journal of Aging Research. 2013;2013:1-8.

2. Nadler, Charlie. 6 Reasons Why Swimming Is Great Exercise for seniors. National Swimming Pool Foundation, 11 Jan 2013. [Internet] Web. 1 Dec 2015 Available from: http://www.nspf.org/en/blog/13-01-11/6_Reasons_Why_Swimming_is_Great_Exercise_for_Seniors.aspx

3. Hawkins HL, Kramer AF, Capaldi D. Aging, exercise and attention. Psychology and Aging. 1992;7(4):643-53.

4. Esther S, Elisabeth MS, Otfried S. A compendium of neuropsychological tests. $3^{\text {rd }}$ ed. New York: Oxford University Press. 2006.

5. Colcombe S, Kramer AF. Fitness effects on the cognitive function of older adults: a meta-analytic study. Psychological Science. 2003;14(2):125-30.

6. AbourezkT, TooleT. Effect of task complexity on the relationship between physical fitness and reaction time in older women. Journal of Aging and Physical Activity 1995;3(3):251-60.

7. Clarkson-Smith L, Hartley AA. Relationships between physical exercise and cognitive abilities in older adults. Psychology and Aging. 1989;4(2):183-9.

8. Hillman $\mathrm{CH}$, Weiss EP, Hagberg JM, Hatfield BD. The relationship of age and cardiovascular fitness to cognitive and motor processes. Psychophysiology. 2002;39(3):303-12.

9. Renaud M, Bherer L, Maquestiaux F. A high level of physical fitness is associ- 
ated with more efficient response preparation in older adults. Journals of Gerontology B. 2010;65(3):317-22.

10. Spirduso WW. Reaction and movement time as a function of age and physical activity level. Journals of Gerontology. 1975;30(4):435-40.
11. Kramer AF, Hahn S, Cohen NJ, et al. Ageing, fitness and neurocognitive function. Nature. 1999;400(6743):418-9.

12. Tarumi T, Zhang R. Cerebral hemodynamics of the aging brain: risk of Alzheimer disease and benefit of aerobic exercise. Front Physiol. 2014;5:6.

Cite this article: Varsha SV. Swimming Helps Elderly Population to Improve Mental Speed and Attention. Int J Clin Exp Physiol. 2018;5(4):200-2. 